\title{
CpG islands around exon 1 in the succinyl-CoA:3-ketoacid CoA transferase (SCOT) gene are hypomethylated even in human and mouse hepatic tissues where SCOT gene expression is completely suppressed
}

\author{
TOSHIYUKI FUKAO ${ }^{1,2}$, GAIXIU ZHANG ${ }^{1}$, NAOKI MATSUO ${ }^{1}$ and NAOMI KONDO ${ }^{1}$ \\ ${ }^{1}$ Department of Pediatrics, Graduate School of Medicine, and ${ }^{2}$ Medical Information Science Division, \\ United Graduate School of Drug Discovery and Medical Information Sciences, Gifu University, Gifu 501-1194, Japan
}

Received November 23, 2009; Accepted January 29, 2010

DOI: $10.3892 / \mathrm{mmr} 00000265$

\begin{abstract}
In ketone body metabolism, hepatocyte-specific silencing of the succinyl-CoA:3-ketoacid CoA transferase (SCOT) gene appears to be physiologically important to avoid a futile cycle in the liver, whereas the SCOT gene is expressed in extrahepatic tissues. It is not possible to explain hepatocyte-specific silencing by cis-elements in the $2.2-\mathrm{kb} 5^{\prime}$ flanking region. The molecular basis of this gene silencing is unknown thus far. In the present study, the methylation status of $\mathrm{CpG}$ islands around exon 1 in the SCOT gene was analyzed by sodium bisulfite treatment and by sequencing of genomic DNA from the HepG2, Chang liver and HeLa human cell lines, and also from mouse liver, heart and kidney cells. Most $\mathrm{CpG}$ dinucleotides in the $\mathrm{CpG}$ island of the human SCOT promoter region were not methylated in the DNA of HeLa and Chang cells, while HepG2 DNA was hypomethylated in this $\mathrm{CpG}$ island. $\mathrm{CpG}$ dinucleotides in the mouse SCOT CpG island were almost completely unmethylated in the liver DNA as well as in the heart and kidney DNA. CpG islands around the promoter region of the SCOT gene were hypomethylated in the DNA from both human HepG2 cells and mouse liver. Hence, methylation status does not contribute to hepatocytespecific SCOT gene silencing.
\end{abstract}

\section{Introduction}

Ketone bodies are important vectors of energy transfer from the liver to extrahepatic tissues, especially when glucose is in short supply (1). Succinyl-CoA:3-ketoacid CoA transferase (SCOT; EC2.8.3.5; locus symbol OXCT) catalyzes the rate-

Correspondence to: Dr Toshiyuki Fukao, Department of Pediatrics, Graduate School of Medicine, Gifu University, 1-1 Yanagido, Gifu, Gifu 501-1194, Japan

E-mail: toshi-gif@umin.net

Key words: succinyl-CoA:3-ketoacid-CoA transferase, OXCT, gene silencing, $\mathrm{CpG}$ island, methylation determining step of ketone body utilization (ketolysis) in extrahepatic tissues. SCOT protein is abundant in the heart, brain and kidney, and has been detected in all extrahepatic tissues tested (2). In extrahepatic tissues, SCOT-activated acetoacetyl-CoA is cleaved to acetyl-CoA by mitochondrial acetoacetyl-CoA thiolase (T2). Acetyl-CoA is then converted to fuel via the Krebs cycle.

SCOT mRNA expression is almost completely suppressed in the human liver, the site of most ketone body synthesis (2). This can be viewed as a mechanism to avoid futile cycling. On the other hand, T2 is abundant in the liver, where it is also involved in ketogenesis and isoleucine catabolism. Notably, rat hepatoma cell lines exhibit various degrees of SCOT protein expression, while expression was scarcely detected in rat hepatocytes (3). Presumably, this may allow hepatoma cells to use ketone bodies as an energy source.

Hereditary SCOT deficiency is one cause of ketoacidosis and, typically, elevated serum levels of ketone bodies are present even when a patient is well nourished and not acutely ill. We previously cloned human SCOT cDNA (4) and the human SCOT gene and reported its structural organization (5). We also previously investigated the basis of SCOT deficiency at the molecular level (5-9).

In previous studies, we investigated the control of SCOT gene expression, especially the mechanism of SCOT gene silencing in hepatic tissue. We recently demonstrated that two GC boxes in the SCOT promoter region are essential for promoter activity, but failed to identify cis-elements as responsible for the complete hepatocyte-specific suppression of the SCOT gene in the 2.2-kb 5' flanking region (10).

Genomic analysis also showed high GC contents in the promoter, in which there are many $\mathrm{CpG}$ sites, both in human and mouse SCOT genes. It is well known that gene expression is affected by epigenetic control. A DNA region with a high level of $\mathrm{CpG}$ methylation in association with a low level of chromatin histone acetylation is inactive for transcription, whereas a DNA region with a low level of $\mathrm{CpG}$ methylation in association with a high level of chromatin histone acetylation is active for transcription. In the present study, we investigated the methylation status of $\mathrm{CpG}$ islands around exon 1 of the 
SCOT gene in both human cell lines and mouse tissues (including the liver) and found that these SCOT CpG islands were, in general, hypomethylated in both human and mouse hepatic DNA.

\section{Materials and methods}

Samples. Genomic DNA was extracted from HeLa, HepG2 and Chang liver cells. Adult mouse genomic DNA was obtained from the heart, kidney and liver. Animal handling and experimentation were carried out in accordance with the guidelines set by the Institutional Animal Care and Use Committee of Gifu University.

Sodium bisulfite treatment. The bisulfite conversion of genomic DNA was performed using a previously described protocol with minor modifications (11-13). Approximately $500 \mathrm{ng}$ of genomic DNA was digested overnight with HindIII, boiled for $1 \mathrm{~min}$, denatured by adding freshly prepared $3 \mathrm{M}$ $\mathrm{NaOH}$ for a final concentration of $0.3 \mathrm{M}$, and incubated at $42^{\circ} \mathrm{C}$ for $30 \mathrm{~min}$. A fresh solution of $3.8 \mathrm{M}$ sodium bisulfite was prepared and adjusted to $\mathrm{pH} 5.0$ with $\mathrm{NaOH}$ and $20 \mathrm{mM}$ hydroquinone by gentle mixing at $37^{\circ} \mathrm{C}$. Final concentrations of $3.4 \mathrm{M}$ sodium bisulfite and $1 \mathrm{mM}$ hydroquinone were added to the denatured DNA for a final volume of $100 \mu \mathrm{l}$. The DNA was gently mixed in this sodium bisulfite/hydroquinone solution, overlaid with mineral oil and incubated at $55^{\circ} \mathrm{C}$ for $6 \mathrm{~h}$. After recovering the aqueous phase from under the oil, the unbound bisulfite was removed from the DNA using microspin S-200HR columns (Pharmacia Biotech). The purified DNA sample was subsequently mixed and incubated with freshly prepared $\mathrm{NaOH}(0.3 \mathrm{M}$ final concentration) at $37^{\circ} \mathrm{C}$ for $20 \mathrm{~min}$. The $\mathrm{NaOH}$ was removed using microspin $\mathrm{S}-200 \mathrm{HR}$ columns, and the flow-through $(<100 \mathrm{ml})$ contained the converted DNA ready for amplification.

PCR conditions. PCR amplifications were carried out in 50- $\mu \mathrm{l}$ reaction mixtures containing 2-8 $\mu \mathrm{l}$ of bisulfite-treated genomic DNA. PCR amplification was performed under the following general conditions: $94^{\circ} \mathrm{C}$ for $5 \mathrm{~min}$, followed by 40 cycles at $94^{\circ} \mathrm{C}$ for $1 \mathrm{~min}, 60^{\circ} \mathrm{C}$ for $1 \mathrm{~min}, 72^{\circ} \mathrm{C}$ for $1 \mathrm{~min}$ and 1 cycle at $72^{\circ} \mathrm{C}$ for $7 \mathrm{~min}$.

For human SCOT CpG islands around exon 1, including the basic promoter region, the following two sets of primers were used: fragment 1, hMETH1 (sense) 5'-GGGTTTTGA ATTTTAGGTTAAGATTTATTT-3'; hMETH11 (antisense) 5'-ACTTTACCTTATACCAAATTA CCCAAATC-3'; and fragment 2, hMETH2 (sense) 5'-GAT TTGGGGTAATTTGGTATAAGGTAAAGT-3'; hMETH22 (antisense) 5'-CCATA ACTA ACCCAACCTCAATTCTA AAC-3'.

For mouse SCOT CpG islands around exon 1, including the basic promoter region, the following two sets of primers were used: fragment 1, mMETH1 (sense) 5'-TTAGTA AGAGATTTTTTAGGTTTTTGGTAA-3'; mMETH11 (antisense) 5'-CCCTACACCTTCAATTTACCTTATACAAA-3'; and fragment 2, mMETH2 (sense) 5'-TTGAAGGTGTAGGGGG GTAAGAGGAAGGTT-3'; mMETH22 (antisense) 5'-CCTT CCCAAAAAC(G/A)TC(G/A)ACCTAAAACC-3'.

The primer positions are shown in Figs. 1 and 3.
Cloning sequencing and analysis of PCR products. Amplified fragments were separated following electrophoresis on a $1 \%(\mathrm{w} / \mathrm{v})$ agarose gel and extracted using a Geneclean II kit (Bio 101, Vista, CA). Isolated PCR fragments were ligated into a pGEM-T Easy vector (Promega) for subcloning. Sequencing was carried out using ABI PRISM ${ }^{\mathrm{TM}}$ Cycle Sequencing kits (Perkin-Elmer Corp., Foster City, CA) and analyzed on an ABI PRISM 3100 Genetic Analyzer (Applied Biosystems). We sequenced ten randomly picked clones after subcloning them into a pGEM-T Easy vector.

\section{Results}

Methylation status in CpG islands around exon 1 in the human SCOT gene. As shown in Fig. 1, more than $80 \mathrm{CpG}$ dinucleotides exist within 1,000 bases. The 5' flanking region of the SCOT gene includes two GC boxes and transcription starting sites, and its GC content is $68.3 \%$. Exon 1 consists of 79 nucleotides and its $\mathrm{GC}$ content is $63.3 \%$. The 5' region of intron 1 ( $\sim 500 \mathrm{bp})$ also has a high GC content of $64.4 \%$. Genomic DNA was extracted from HeLa, Chang liver and HepG2 cells. This region was divided into two fragments, which were analyzed by bisulfite sequencing.

Fig. 2 shows the percentage of $\mathrm{CpG}$ methylation at individual $\mathrm{CpG}$ dinucleotides (the numbering of $\mathrm{CpG}$ dinucleotides is as shown in Fig. 1) in these cell lines. In general, this region was almost entirely composed of non-methylated DNA in the HeLa and Chang liver cells. CpG dinucleotides no. 63-83 were rather more methylated than the other dinucleotides in HepG2 DNA. DNA from HepG2 cells was the most methylated among these three cell lines, and was regarded as hypomethylated in this region.

Methylation status in $C p G$ islands around exon 1 in the mouse SCOT gene. As shown in Fig. 3, more than $80 \mathrm{CpG}$ dinucleotides exist within 960 bases around exon 1 as well as the human SCOT gene. Genomic DNA was extracted from mouse kidney, heart and liver tissues. This region was divided into two fragments, which were analyzed by bisulfite sequencing. Unexpectedly, the DNA from mouse liver was completely unmethylated in these $81 \mathrm{CpG}$ dinucleotides, as was the DNA from mouse kidney and heart.

\section{Discussion}

Liver-specific SCOT gene silencing appears to be physiologically important. This silencing is observed in humans and mice. Previously, we studied the molecular basis of this liver-specific SCOT gene silencing. In the present study, we compared the methylation status of the $\mathrm{CpG}$ islands around exon 1 of the human SCOT gene in two hepatoma cell lines (HepG2 and Chang liver cells) and in the HeLa cervical cancer cell line. We showed that SCOT mRNA and protein were detectable in Chang liver cells and the HeLa cervical cancer cell line, but not in the HepG2 cell line, indicating that the latter maintains the characteristics of liver cells in ketone body metabolism (10). In general, the $\mathrm{CpG}$ islands around exon 1 were non-methylated in HeLa and Chang liver cells and were hypomethylated (up to $60 \%$ ) in the HepG2 cell line. Since SCOT gene silencing was almost complete, the difference among these cell lines 
Hind III

AAGCTTGGCGGGAGATCCTCCCGCGGCCGGA GGGCCCTGAACCCTAGGCCAAGACTCATCCACAACCTAGGGGGAGGCGGCC

ACCAGCGCCCCATCGAAGCTAAAGGGCTGACAGAAGGGCTGTAGCGGCAAGTGTGCGCCAACACAGCTGCACACGCCCAGAC ACCTGAGGCGCTGAGAGGAACTTCCCCGCGATCCGCCCCGGCTGCACAGAGCCTCTTCTCCCCGCCGGGGCCCCGCCCAGCCT CACTTCTTTTAAAAGCAGCAGCCAGCAGCGCGCCGGTGGGCGTCGGGCTCGAAGGCCGGGAGGACGTCATCGACGCGCTG GC box 2

TCGAGCCTCCAGCCCGCCCGGGTTCCTTCGCAGTCGCGCACCGACGCTCAAACGCGCGCTCCAACCCGCAGCCTCCTCCTGC CTCACCGCCCGAAG

Initiator MET ATGGCG GCTCTCAAAATCCTCTCCTCCG GGTTCG GGCTCTGCG GCCTATGCCCG C GGATCTGGGGCAACCTGGTACAAG

Intron 1

GTAAAGTGCGGGGGAAGAGGAAGGCCATGGGCAGGAAGAACGTGGACCAAACCCTGAGTGGTGCCCGCGTTTTCTTCTCTGC CCCTACCCGCTCTCTCCAGCCTGGGATCTGGCATCCGTGGCGCGGTGCCCTGGTCACGGACACGCGGCGACGCGTTCCTGGCC TCGCCGGCCCCTCGGATGACCGCCTTTGCTTGGTTCTGCAGTCGCTTCCCTGATGGAGCGAGACGCGATAAAGAAGCGCGCGG AGAGGCTGAGAAGGGCGGGAGGACTCCTGGCAGCGCTCCCGAGCCCTTTGGCGCTCGTCGGCTTCCCTCCTGGCACCCGCC CTTGGATAGGGACTCTGAGGGTCTGAACGGGGGGAGGAGGGCGCCAGTATATGGAGGTTTAGGAGTGAGGCAACAGCGGGTA

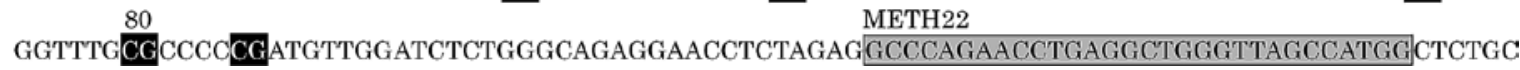

Figure 1. CpG islands around exon 1 of the human SCOT gene. CpGs are indicated by a black background. CpG dinucleotides are numbered from a 5 ' to 3 orientation. Two GC boxes and major transcription starting points are indicated. The nucleotides in exon 1 are indicated by italicized characters. The positions of the primers used in the PCR after bisulfite treatment are indicated by a grey background.

\begin{tabular}{|c|c|c|c|c|c|c|c|c|c|c|c|c|c|c|}
\hline \multirow[b]{2}{*}{ CpG number } & \multicolumn{14}{|l|}{$5^{\prime}$} \\
\hline & 1 & 2 & 3 & 4 & 5 & 6 & 7 & 8 & 9 & 10 & 11 & 12 & 13 & 14 \\
\hline HepG2 & 10 & 0 & 10 & 10 & 30 & 0 & 10 & 0 & 10 & 0 & 0 & 10 & 10 & 10 \\
\hline Chang & 10 & 20 & 0 & 0 & 0 & 0 & 0 & 0 & 0 & 0 & 0 & 0 & 0 & 0 \\
\hline HeLa & 0 & 0 & 10 & 0 & 0 & 0 & 0 & 0 & 10 & 0 & 0 & 10 & 0 & 0 \\
\hline CpG number & 15 & 16 & 17 & 18 & 19 & 20 & 21 & 22 & 23 & 24 & 25 & 26 & 27 & 28 \\
\hline HepG2 & 30 & 10 & 30 & 10 & 10 & 10 & 10 & 10 & 10 & 0 & 0 & 10 & 10 & 10 \\
\hline Chang & 0 & 0 & 0 & 0 & 0 & 0 & 0 & 0 & 0 & 0 & 0 & 0 & 0 & 0 \\
\hline HeLa & 10 & 0 & 0 & 0 & 0 & 0 & 0 & 0 & 0 & 0 & 0 & 0 & 0 & 10 \\
\hline CpG number & 29 & 30 & 31 & 32 & 33 & 34 & 35 & 36 & 37 & 38 & 39 & 40 & 41 & 42 \\
\hline HepG2 & 60 & 0 & 10 & 40 & 30 & 30 & 10 & 30 & 30 & 60 & 10 & 40 & 0 & 0 \\
\hline Chang & 0 & 0 & 0 & 0 & 0 & 0 & 0 & 0 & 0 & 0 & 0 & 0 & 0 & 0 \\
\hline HeLa & 0 & 0 & 0 & 0 & 0 & 0 & 0 & 0 & 0 & 0 & 0 & 0 & 0 & 0 \\
\hline CpG number & 43 & 44 & 45 & 46 & 47 & 48 & 49 & 50 & 51 & 52 & 53 & 54 & 55 & 56 \\
\hline HepG2 & 0 & 0 & 0 & 0 & 0 & 0 & 0 & 40 & 10 & 0 & 0 & 30 & 10 & 20 \\
\hline Chang & 0 & 0 & 0 & 0 & 10 & 10 & 0 & 0 & 0 & 0 & 0 & 0 & 0 & 0 \\
\hline HeLa & 0 & 0 & 0 & 0 & 0 & 0 & 0 & 0 & 10 & 0 & 0 & 0 & 0 & 0 \\
\hline CpG number & 57 & 58 & 59 & 60 & 61 & 62 & 63 & 64 & 65 & 66 & 67 & 68 & 69 & 70 \\
\hline HepG2 & 0 & 20 & 10 & 10 & 0 & 30 & 30 & 40 & 40 & 30 & 40 & 40 & 40 & 50 \\
\hline Chang & 0 & 0 & 0 & 0 & 0 & 0 & 0 & 0 & 0 & 0 & 0 & 0 & 0 & 0 \\
\hline HeLa & 0 & 0 & 0 & 0 & 0 & 0 & 0 & 0 & 0 & 0 & 0 & 0 & 0 & 0 \\
\hline CpG number & 71 & 72 & 73 & 74 & 75 & 76 & 77 & 78 & 79 & 80 & 81 & & & \\
\hline HepG2 & 40 & 40 & 30 & 20 & 40 & 30 & 40 & 30 & 40 & 40 & 40 & & & \\
\hline Chang & 0 & 0 & 0 & 0 & 0 & 0 & 0 & 0 & 0 & 0 & 0 & & & \\
\hline HeLa & 0 & 0 & 0 & 0 & 0 & 0 & 0 & 0 & 0 & 0 & 0 & & & \\
\hline
\end{tabular}

Figure 2. Methylation status in the CpG island around exon 1 of the human SCOT gene. Ten clones of each PCR fragment after bisulfite treatment were sequenced. Percentages of methylated $\mathrm{CpG}$ dinucletides are shown. The number of $\mathrm{CpG}$ dinuceltides is shown in Fig. 1. 


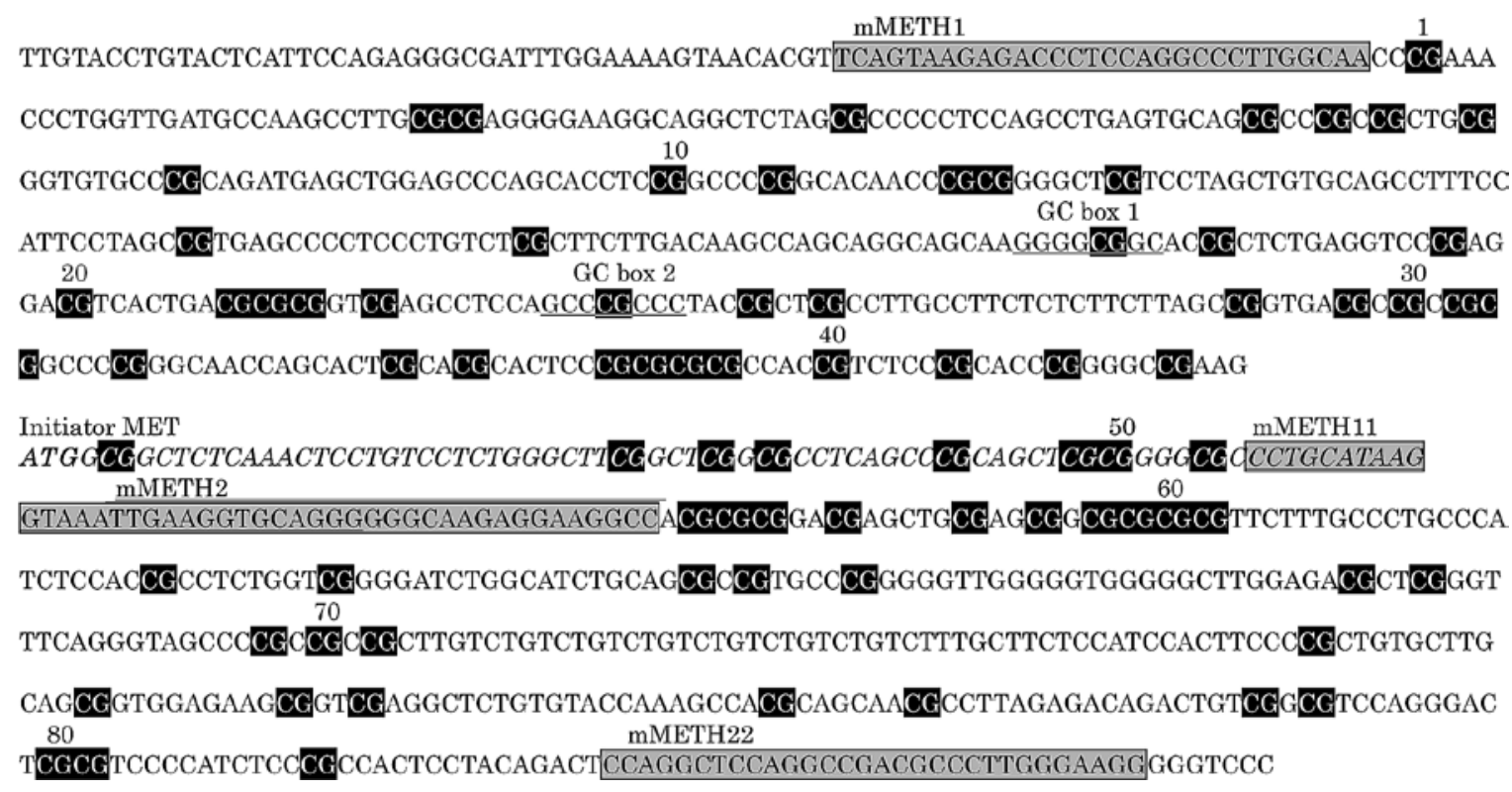

Figure 3. CpG islands around exon 1 of the mouse SCOT gene. CpGs are indicated by a black background. CpG dinucleotides are numbered from a 5 ' to 3 orientation. Two GC boxes and major transcription starting points are indicated. The nucleotides in exon 1 are indicated by italicized characters. The positions of the primers used in PCR after bisulfite treatment are indicated by a grey background.

was likely due to the mechanism of gene silencing. Since hepatocyte-specific silencing is observed not only in humans, but also in mice, we analyzed the methylation status around exon 1 of the mouse SCOT gene. Most CpG dinucleotides in the $\mathrm{CpG}$ islands around exon 1 of the mouse SCOT gene were not methylated in the hepatic DNA or in the DNA from the heart and kidney. Hence, the methylation status around exon 1 of the human and mouse SCOT genes does not contribute to hepatocyte-specific SCOT gene silencing.

In normal hepatocytes, SCOT gene expression is almost completely suppressed, whereas all extrahepatic tissues tested exhibited SCOT expression to various degrees, as follows: myocardium $>$ brain kidney adrenal glands $>$ other tissues (2). The activation of acetoacetate to acetoacetyl-CoA by SCOT is essential for the use of ketone bodies as an energy source in extrahepatic tissues (1). The absence of SCOT in hepatocytes is an important element in energy metabolism, suppressing ketolysis in the liver that might otherwise create a futile cycle and interfere with the efficiency of ketogenesis. Another important aspect of SCOT gene regulation is that some hepatoma cell lines have detectable SCOT expression (3). Such dysregulation benefits tumor cells since there is a relationship between SCOT expression and their growth rate. Hence, this hepatocyte-specific suppression should be programmed and conserved in mammalians.

We recently demonstrated that two GC boxes in the SCOT promotor region are essential for promotor activity, but failed to identify the specific cis-elements responsible for the complete silencing of the SCOT gene in hepatic tissues in the 2.2-kb $5^{\prime}$ flanking region (10). One possibility is that liver-specific elements, such as strong suppressors or silencers in the SCOT gene, may lie outside of the 2.2-kb 5' flanking region. Alternatively, other mechanisms of gene silencing, such as methylation and siRNA, may be involved in hepatocyte-specific SCOT gene silencing.
DNA methylation is an evolutionally conserved mechanism for the regulation of gene expression in mammals. Tissue- and disease-specific de novo methylation events are observed during somatic cell development/differentiation. Once established, DNA methylation patterns are thought to be stable. Generally, cytosine residues in $\mathrm{CpG}$ are methylated in the genome, especially within non-coding DNA, introns and repetitive sequences. Most $\mathrm{CpG}$ clusters, called $\mathrm{CpG}$ islands, which are frequently found in the proximal promoter regions of many genes, are unmethylated during normal cell development. However, there are exceptions, such as imprinted genes, genes on the inactive $\mathrm{X}$ chromosome and tissue-specific differentially methylated genes. DNA methylation plays a role in the regulation of tissue-specific gene expression $(14,15)$. Comparative analysis between mice and humans suggests that some, but not all, tissue-specific differentially methylated regions are conserved (16).

At least $5 \%$ of $15,500 \mathrm{CpG}$ islands in the mouse are differentially methylated (17), and 50 tissue-specific differentially methylated regions have been identified. The majority of the tissue-specific differentially methylated regions are associated with 5 ' promoter $\mathrm{CpG}$ islands, and may play important roles in establishing or maintaining gene silencing during or after tissue differentiation.

Here, we investigated whether the methylation status of $\mathrm{CpG}$ islands around exon 1, including the SCOT gene promoter in the liver, is different from other tissues which express the SCOT gene, such as heart and kidney tissue. We clearly demonstrated that most $\mathrm{CpG}$ dinucleotides in $\mathrm{CpG}$ islands around exon 1 of the SCOT gene were hypomethylated or non-methylated in DNA from human and mouse hepatic tissues, as well as DNA from SCOT-expressed cells and tissues. The tissue-specific-expressed human $\beta$-globin (18) and $\alpha 2$ (1) collagen (19) genes were found to have $\mathrm{CpG}$ islands that remain unmethylated in tested tissues regardless of 
expression. Since many $\mathrm{CpG}$ islands are located at genes that have a tissue-restricted expression pattern, it follows that $\mathrm{CpG}$ islands remain methylation-free, even when their associated gene is silent (20). In other words, a cluster of hypomethylated $\mathrm{CpG}$ dinucleotides may be a common characteristic of $\mathrm{CpG}$ islands, while some tissue-specific differentially methylated $\mathrm{CpG}$ islands have also been identified. Hence, the SCOT gene has a typical $\mathrm{CpG}$ island that remains un- or hypomethylated. Further analysis is needed to understand liver-specific silencing of the SCOT gene.

\section{Acknowledgements}

We thank N. Sakaguchi for technical assistance. This study was supported in part by a Grant-in-Aid for Scientific Research from the Ministry of Education, Science, Sports and Culture of Japan, and by the Health and Labor Science Research Grants for Research on Intractable Diseases and on Research on Children and Families from The Ministry of Health, Labor and Welfare of Japan.

\section{References}

1. Mitchell GA and Fukao T: Inborn errors of ketone body catabolism. In: Molecular and Metabolic Bases of Inherited Disease. 8th edition. Scriver CR, Beaudet AL, Sly WS and Valle D (eds). McGraw-Hill Inc., New York, pp2327-2356, 2001.

2. Fukao T, Song X-Q, Mitchell GA, Yamaguchi S, Sukegawa K, Orii $\mathrm{T}$ and Kondo $\mathrm{N}$ : Enzyme of ketone body utilization in human tissues: protein and messenger RNA levels of succinyl-Coenzyme A (CoA):3-ketoacid CoA transferase and mitochondrial and cytosolic acetoacetyl-CoA thiolases. Pediatr Res 42: 498-502, 1997.

3. Zhang WW, Lindahl R and Churchill P: Regulation of succinyl coenzyme A: acetoacetyl coenzyme A transferase in rat hepatoma cell lines. Cancer Res 50: 5858-5862, 1990.

4. Kassovska-Bratinova S, Fukao T, Song X-Q, Duncan A, Chen HS, Robert M-F, Perez-Cerda C, Ugarte M, Chartrand P, Vobecky S, Kondo N and Mitchell GA: Succinyl-CoA:3-ketoacid CoA transferase (SCOT): Human SCOT cDNA cloning and chromosomal mapping and mutation detection in a SCOT-deficient patient. Am J Hum Genet 59: 519-528, 1996.

5. Fukao T, Mitchell GA, Song X-Q, Nakamura $H$, Kassovska-Bratinova S, Orii KE, Wraith JE, Besley G, Wanders RJA, Niezen-Koning KE, Berry GT, Palmieri M and Kondo N: Succinyl-CoA:3-ketoacid CoA transferase (SCOT) cloning of the human SCOT gene tertiary structural modeling of the human SCOT monomer and characterization of three pathogenic mutations. Genomics 68: 144-151, 2000.

6. Fukao T, Shintaku H, Kusubae R, Zhang X-Q, Nakamura K, Kondo $\mathrm{M}$ and Kondo N: Patients homozygous for the T435N mutation of succinyl-CoA:3-ketoacid CoA transferase (SCOT) do not show permanent ketosis. Pediatr Res 56: 858-863, 2004.
7. Fukao T, Sakurai S, Rolland M-O, Zabot M-T, Schulze A, Yamada $\mathrm{K}$ and Kondo N: A 6-bp deletion at the splice donor site of the first intron resulted in aberrant splicing using a cryptic splice site within exon 1 in a patient with succinyl-CoA:3ketoacid CoA transferase (SCOT) deficiency. Mol Genet Metab 89: 280-282, 2006

8. Fukao T, Kursula P, Owen EP and Kondo N: Identification and characterization of a temperature-sensitive R268H mutation in the human succinyl-CoA:3-ketoacid CoA transferase (SCOT) gene. Mol Genet Metab 92: 216-221, 2007.

9. Yamada K, Fukao T, Zhang G, Sakurai S, Ruiter JPN, Wanders RJA and Kondo N: Single-base substitution at the last nucleotide of exon 6 (c $671 \mathrm{G}>\mathrm{A}$ ), resulting in the skipping of exon 6 and exons 6 and 7 in human Succinyl-CoA:3-ketoacid CoA transferase (SCOT) gene. Mol Genet Metab 90: 291-297, 2007.

10. Orii KE, Fukao T, Song X-Q, Mitchell GA and Kondo N: Liver-specific silencing of the human gene encoding succinylCoA:3-ketoacid CoA transferase. Tohoku J Exp Med 215: 227-236, 2008

11. Clark SJ, Harrison J, Paul CL and Frommer M: High sensitivity mapping of methylated cytosines. Nucleic Acids Res 22: 2990-2997, 1994.

12. Warnecke PM, Stirzaker C, Song J, Grunau C, Melki JR and Clark SJ: Identification and resolution of artifacts in bisulfite sequencing. Methods 27: 101-107, 2002.

13. Tomatsu S, Orii KO, Islam MR, Shah GN, Grubb JH, Sukegawa K, Suzuki Y, Orii T, Kondo N and Sly WS: Methylation patterns of the human beta-glucuronidase gene locus: boundaries of methylation and general implications for frequent point mutations at CpG dinucleotides. Genomics 79: 363-375, 2002.

14. Futscher BW, Oshiro MM, Wozniak RJ, Holtan N, Hanigan CL, Duan $\mathrm{H}$ and Domann FE: Role for DNA methylation in the control of cell type specific maspin expression. Nat Genet 31 : 175-179, 2002

15. Ching TT, Maunakea AK, Jun P, Hong C, Zardo G, Pinkel D, Albertson DG Fridlyand J, Mao JH, Shchors K, Weiss WA and Costello JF: Epigenome analyses using BAC microarrays identify evolutionary conservation of tissue-specific methylation of SHANK3. Nat Genet 37: 645-651, 2005.

16. Nagase $\mathrm{H}$ and Ghosh S: Epigenetics: differential DNA methylation in mammalian somatic tissues. FEBS J 275: 1617-1623, 2008.

17. Song F, Smith JF, Kimura MT, Morrow AD, Matsuyama T, Nagase $\mathrm{H}$ and Held WA: Association of tissue-specific differentially methylated regions (TDMs) with differential gene expression. Proc Natl Acad Sci USA 102: 3336-3341, 2005.

18. Bird AP, Taggart MH, Nicholls RD and Higgs DR: Non-methylated CpG-rich islands at the human alpha-globin locus: implications for evolution of the alpha-globin pseudogene. EMBO J 6: 999-1004, 1987.

19. McKeon C, Ohkubo H, Pastan I and de Crombrugghe B: Unusual methylation pattern of the alpha 2(1) collagen gene. Cell 29: 203-210, 1982

20. Bird A: DNA methylation patterns and epigenetic memory. Genes Dev 16: 6-21, 2002. 